\title{
Trans-sonic cusped shaped, periodic waves and solitary waves of the electrostatic ion-cyclotron type
}

\author{
J. F. McKenzie ${ }^{1,2,3}$ and T. B. Doyle ${ }^{2}$ \\ ${ }^{1}$ Max-Planck-Institute for Solarsystems Research, Katlenburg-Lindau, Germany \\ ${ }^{2}$ School of Physics \& School of Mathematical and Statistical Sciences, Univ. of KwaZulu-Natal, Durban, 4041, South Africa \\ ${ }^{3}$ Department of Physics, University of Alabama at Huntsville, USA
}

Received: 3 June 2004 - Revised: 23 August 2004 - Accepted: 6 September 2004 - Published: 24 September 2004

Part of Special Issue "Nonlinear plasma waves-solitons, periodic waves and oscillations"

\begin{abstract}
By adopting an essentially fluid dynamic viewpoint we derive the wave structure equation for stationary, fully nonlinear, electrostatic, ion-cyclotron waves. The existence of two fundamental constants of the motion, namely, conservation of momentum flux parallel to the ambient magnetic field, and energy flux parallel to the direction of wave propagation, enables the wave structure equation to be reduced to a first order differential equation, which has solutions that are physically transparent. The analysis shows that sufficiently oblique waves, propagating at sub-ion acoustic speeds, form soliton pulse-like solutions whose amplitudes are greatest for perpendicular propagation. Waves that propagate supersonically have periodic cnoidal waveforms, which are asymmetric about the compressive and rarefactive phases of the wave. It is also shown that there exist critical driver fields for which the end point of the compressive phase goes sonic (in the wave frame), with the consequence that the wave form develops a cusp. It is possible that this trans-sonic, choked flow feature provides a mechanism for the "spiky" waveforms observed in auroral electric field measurements.
\end{abstract}

\section{Introduction}

In electrostatic, ion-cyclotron waves, with frequencies $(\omega)$ very much less than the electron gyrofrequency $\left(\Omega_{e}\right)$ the electrons are constrained to move along the ambient magnetic field $\boldsymbol{B}_{o}=B_{o}(\cos \theta, 0, \sin \theta)$. Furthermore, if $\omega<<$ $\omega_{p i}$ (where $\omega_{p i}$ is the ion plasma frequency) quasi-charge neutrality prevails, so that for wave propagation parallel to the $\mathrm{x}$-axis and at angle $\theta$ to $\boldsymbol{B}_{o}$, continuity requires that the $\mathrm{x}$ components of the electron and ion velocities are very nearly equal. The linearized equations of motion for any perturbed

Correspondence to: T. B. Doyle

(doyle@ukzn.ac.za) wave variable, such as for example the potential, $\phi$, readily yield the wave equation of the system, namely:

$$
\left[\left(\frac{\partial^{2}}{\partial t^{2}}+\Omega_{x}^{2}\right)\left(\frac{\partial^{2}}{\partial t^{2}}-c^{2} \frac{\partial^{2}}{\partial x^{2}}\right)+\Omega_{z}^{2} \frac{\partial^{2}}{\partial t^{2}}\right] \phi=0,
$$

where

$$
\boldsymbol{\Omega}=\Omega(\cos \theta, 0, \sin \theta), \quad \Omega=\frac{e B_{o}}{m_{i}},
$$

and

$c^{2}=\frac{\left(\gamma_{e} k T_{e}+\gamma_{i} k T_{i}\right)}{m_{i}}$.

Here $c$ is the ion-acoustic speed, based on the electron and ion temperatures, $T_{e, i}$, their adiabatic indices $\gamma_{e, i}$, and the ion mass $m_{i}$. In this wave equation the transverse cyclotron waves are coupled to the longitudinal "sound" waves through the motional electric field and quasi charge neutrality, both of which effects are embedded in the $\Omega_{z}^{2} \partial^{2} / \partial t^{2}$ term. The corresponding dispersion equation for plane harmonic waves (varying as exp $i(\omega t-k x)$ ) follows immediately (also see e.g. Stix, 1992) and is given by

$c^{2} k^{2}=\omega^{2} \frac{\left(\omega^{2}-\Omega^{2}\right)}{\left(\omega^{2}-\Omega^{2} \cos ^{2} \theta\right)}$.

Thus low frequency waves propagate at the phase speed, $c \cos \theta$, before encountering a resonance at $\omega=\Omega \cos \theta$, and the high frequency branch exhibits a cut-off at the gyrofrequency $\Omega$ before propagating isotropically at the ion-acoustic speed, $c$, for $\omega>>\Omega$. In the "gap", i.e. $c \cos \theta<\omega / k<c$ or, more generally $\Omega \cos \theta<\omega<\Omega$, the wave is evanescent, with the stationary wave version of Eq. (4), obtained by putting $\omega=U k$, yielding

$k^{2}=\frac{\Omega^{2}}{U^{2}} \frac{\left(U^{2}-c^{2} \cos ^{2} \theta\right)}{\left(U^{2}-c^{2}\right)}$,

showing that $k^{2}<0$, if the stationary wave speed $U$ falls in the "gap". In such a region the inclusion of nonlinear 


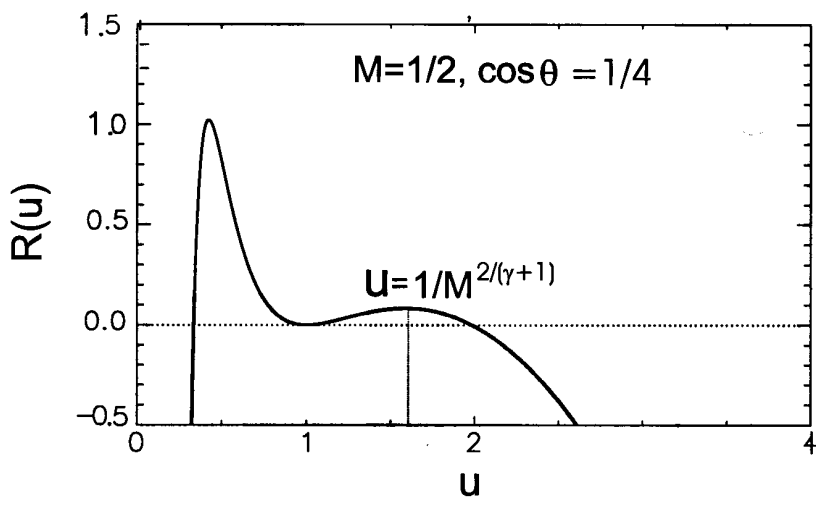

Fig. 1. $R(u)$ as a function of $u$ for $\gamma=2$ and $1>M>\cos \theta$. The amplitude of the soliton is determined by the zero of $R(u)$ on the compression branch of the curve $(u<1)$.

terms, which allow for steepening to balance dispersion, solitons may be formed. The properties of these nonlinear waves have been studied by, for example, Temerin et al. (1979), Yu et al. (1980), and Jovanic and Shukla (2000). Although these waves are interesting in their own right, their properties may explain certain features observed in auroral electric fields. Presumably, with this in mind, Reddy et al. (2002) revisited the problem and formulated a fully nonlinear treatment of these waves. In their formulation the wave structure equation is a rather complicated second order differential equation for the electrostatic potential $\phi$. Nevertheless, they were able to show (numerically) that for supersonic wave speeds and sufficiently large initial perturbations, or driving electric fields, it was possible to construct the type of periodic, spiky waveforms that have been observed in auroral electric fields by Viking (Andre et al., 1987) and FAST (Ergun et al., 1998). However, this numerical study does not reveal the underlying mechanism which can give rise to this curious type of waveform. More recently McKenzie (2004) has shown that, by making use of the constants of the motion, the wave structure equation can be reduced to a first order differential equation. This more physically transparent form shows quite clearly that supersonic waves are periodic, and that in the compressive phase of the wave the flow is driven towards the sonic point. In fact, there exists a critical driving field at which the end point of the compressive phase becomes sonic and a cusp shaped waveform develops.

In this paper we extend this work. We show that subsonic solitons propagate at all angles $\theta>\cos ^{-1}(U / c)$, in contrast to the prediction of the weakly nonlinear KdV treatment, and that their amplitudes, for a given Mach number $(M=U / c)$, are greatest for perpendicular propagation (see Fig. 2). We also show analytically (through a geometrical interpretation of the wave structure equation) how cusped shaped waveforms are generated in trans-sonic choked flow conditions.

\section{The structure equation for nonlinear stationary EICA waves}

Here we provide a brief description of a new formulation of fully nonlinear, stationary low frequency $\left(\omega<<\Omega_{e}\right)$, electrostatic waves in a magnetized plasma. In this fluid dynamic approach the emphasis is placed on the nature of the proton flow, that is to say, whether it is "subsonic" or "supersonic", with the consequence that the crucial role played by the sonic point is rendered manifest. In this picture the electrons are treated as massless $\left(\omega<<\Omega_{e}\right)$ and are thus constrained to move along the magnetic field $\boldsymbol{B}_{o}=B_{o}(\cos \theta, 0, \sin \theta)$, so that, in the wave frame, the electric field $\boldsymbol{E}$, which is curl free from Faraday, is given by

$\boldsymbol{E}=\left(-\frac{1}{e n_{e}} \nabla_{x} \boldsymbol{p}_{e}, U \boldsymbol{B}_{o z}, 0\right)$

in which $\boldsymbol{p}_{e}\left(n_{e}\right)$ is the electron pressure (density), $U \boldsymbol{B}_{o z}$ is the constant motional electric field $E_{y}$, and $U$ is the wave speed. If we assume quasi-charge neutrality, i.e. $n_{e} \doteqdot n_{i}=$ $n$, which is valid for wave frequencies very much less than the ion plasma frequency, then in the wave frame the longitudinal components of the ion and electron velocities are very nearly equal $\left(u_{e x} \simeq u_{i x}\right)$ by virtue of continuity, which for the ions, may be written

$m_{i} n_{i} u_{i x}=m_{i} n_{i o} U \equiv M_{i}$.

In the wave frame the equation of motion for the ions is,

$m_{i} n_{i} \boldsymbol{u}_{i x} \frac{d \boldsymbol{u}_{i}}{d x}=e n_{i}\left(\boldsymbol{E}+\boldsymbol{u}_{i} \times \boldsymbol{B}_{o}\right)-\nabla \boldsymbol{p}_{i}$.

Integrating the scalar product of this equation with $\boldsymbol{B}_{o}$ yields conservation of momentum flux parallel to the ambient magnetic field in the form,

$\cos \theta P\left(u_{i x}\right)+\sin \theta M_{i} u_{i z}=$ const,

in which

$P\left(u_{i x}\right)=M_{i} u_{i x}+\left(p_{e}+p_{i}\right)$.

The first term on the left hand side of Eq. (9) is the component of the longitudinal momentum flux, dynamic pressure plus thermal pressures, along $\boldsymbol{B}_{o}$, and the second term is the component of the transverse Reynolds' stress parallel to $\boldsymbol{B}_{o}$. The third constant of the motion is energy flux conservation which follows from the integral of scalar product of $\boldsymbol{u}_{i}$ with the equation of motion to give

$M_{i}\left[\frac{1}{2}\left(u_{i x}^{2}+u_{i y}^{2}+u_{i z}^{2}\right)+w\right]-U P\left(u_{i x}\right)=$ const,

in which

$w=\left[\frac{\gamma_{e} p_{e}}{\left(\gamma_{e}-1\right)}+\frac{\gamma_{i} p_{i}}{\left(\gamma_{i}-1\right)}\right] \frac{1}{m_{i} n_{i}}$.

Here the first term on the left hand side Eq. (11) is the flux of kinetic energy plus enthalpy $(w)$, and the term $-U P_{i x}$ represents the rate of working of the motional electric field. 

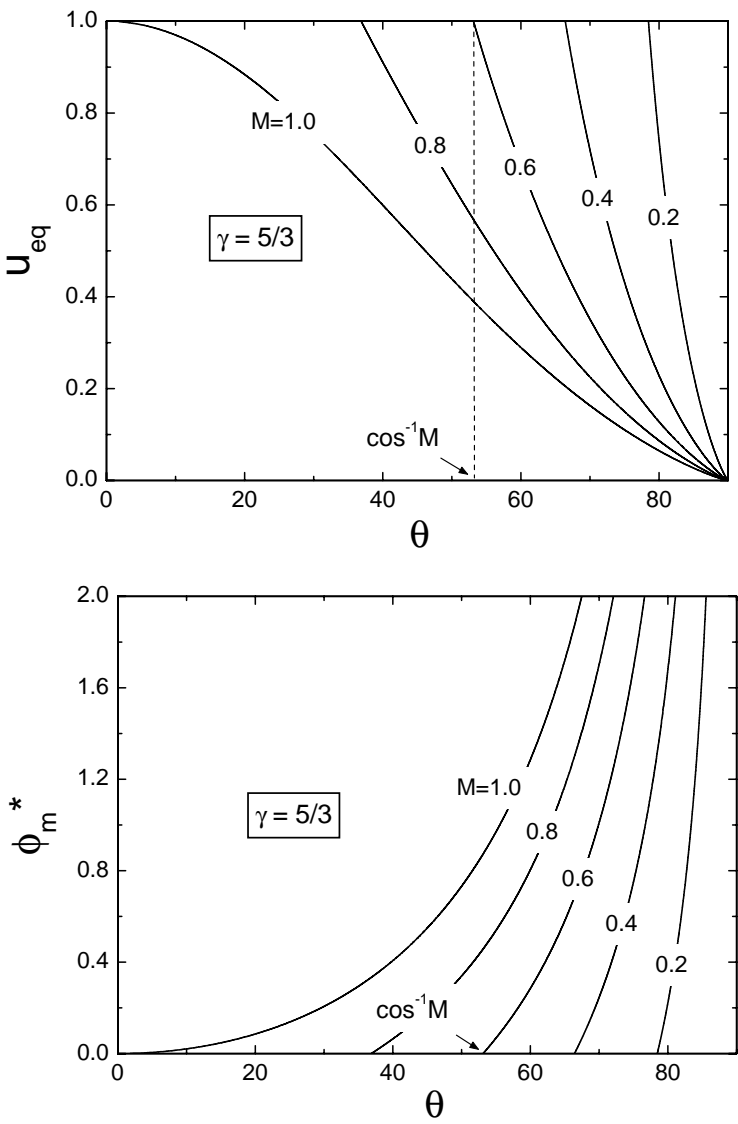

Fig. 2. (a) The strength of compressive solitons, $u_{e q}$, as a function of the obliquity angle $\theta$, for $\gamma=5 / 3$, and for various $M \leqq 1$. (b) The equivalent reduced maximum potential $\phi_{m}^{*} \equiv$ $\left(e \phi_{m}\right) /\left(\frac{5}{2} k T_{e}\right)=\left(1 /\left(u_{e q}^{(\gamma-1)}-1\right)\right.$ for various $M \leqslant 1$.

We have assumed adiabatic flow for each species, i.e. $p_{e, i} \propto$ $n_{e, i}^{\gamma_{e, i}} \propto u_{e, i x}^{-\gamma_{e, i}}$. It is convenient to define the "longitudinal" Bernoulli-like energy density as

$\varepsilon\left(u_{i x}\right)=\frac{1}{2} u_{i x}^{2}+w\left(u_{i x}\right)$.

Note the "thermodynamic" relation

$d \varepsilon=u_{i x} d P$

which, in fact, has been used in obtaining the conservation form, Eq. (11). Thus with $u_{i z}$ given in terms of $u_{i x}$ through momentum conservation (9), and $u_{i y}$, in terms of $u_{i x}$ and its derivative through the $\mathrm{x}$-component of the equation of motion, namely:

$\Omega u_{i y} \sin \theta=\left(u_{i x}-\frac{c^{2}}{u_{i x}}\right) \frac{d u_{i x}}{d x}$,

where $c$ is the ion-acoustic speed given by,

$c^{2}=\frac{\left(\gamma_{e} p_{e}+\gamma_{i} p_{i}\right)}{m_{i} n}$

the energy Eq. (11) is a first order differential equation for the longitudinal flow speed $u_{i x}$ in the wave. In normalized form, therefore, the wave structure is governed by the differential equation,

$$
\begin{aligned}
& \frac{1}{2}\left\{l\left[1-\frac{1}{M_{e}^{2} u^{\left(\gamma_{e}+1\right)}}-\frac{1}{M_{i}^{2} u^{\left(\gamma_{i}+1\right)}}\right] u \frac{d u}{d x}\right\}^{2}= \\
& \sin ^{2} \theta[P(u)-e(u)]-\frac{1}{2} \cos ^{2} \theta P(u) \equiv R(u)
\end{aligned}
$$

in which $l=U / \Omega, u=u_{i x} / U$, and $P(u)$ and $e(u)$ are, respectively, the normalized Bernoulli momentum and energy functions, given by

$P(u)=u-1+\frac{1}{\gamma_{e} M_{e}^{2}}\left(\frac{1}{u^{\gamma_{e}}}-1\right)+\frac{1}{\gamma_{i} M_{i}^{2}}\left(\frac{1}{u^{\gamma_{i}}}-1\right)$,

and

$$
\begin{aligned}
e(u) & =\frac{1}{2}\left(u^{2}-1\right)+\frac{1}{\left(\gamma_{e}-1\right) M_{e}^{2}}\left(\frac{1}{u^{\gamma_{e}-1}}-1\right) \\
& +\frac{1}{\left(\gamma_{i}-1\right) M_{i}^{2}}\left(\frac{1}{u^{\gamma_{i}-1}}-1\right),
\end{aligned}
$$

in which the Mach numbers are given by

$$
M_{e, i}^{2}=\frac{m_{i} U^{2}}{\gamma_{e, i} k T_{e, i}}
$$

In the special case $\gamma_{e}=\gamma_{i}$ the collective ion-acoustic Mach number $M$ is given by

$\frac{1}{M^{2}}=\frac{1}{M_{e}^{2}}+\frac{1}{M_{i}^{2}}$

Note the existence of possible equilibrium points $(d u / d x=$ 0 ) at the roots of $R(u)=0$, and critical points $(d u / d x=\infty)$ where the longitudinal flow speed equals the ion acoustic speed $\left(u_{i x}=c\right)$. Here $R(u)$ represents the change in the total energy of the system at any point (minus the kinetic energy associated with $\frac{1}{2} u_{i y}^{2}$ ) and will be referred to as the total energy function to distinguish it from the Bernoulli energy function $e(u)$, which represents the change in the longitudinal energy density given by Eq. (19).

\section{Periodic and soliton solutions of the wave structure equation}

In general $\gamma_{e} \neq \gamma_{i}$, for example, isothermal electrons $\left(\gamma_{e}=\right.$ 1) and adiabatic protons $\left(\gamma_{i}=5 / 3\right)$ as in the solar wind. However, we do not expect any qualitative difference by assuming $\gamma_{e}=\gamma_{i}=\gamma$ in what follows, since the Bernoulli momentum and energy functions, whose behaviour determines the total energy function $R(u)$, always exhibit a minimum at the sonic point. The wave Eq. (17) admits either soliton or periodic solutions depending on the behaviour of the total energy function $R(u)$, which possesses three extrema, namely: one at $u=1$, the initial point, which is a double zero of $R(u)$, another at $u=1 / M^{[2 / \gamma+1]}$ (for $\gamma_{e}=\gamma_{i}=\gamma$ ), which 


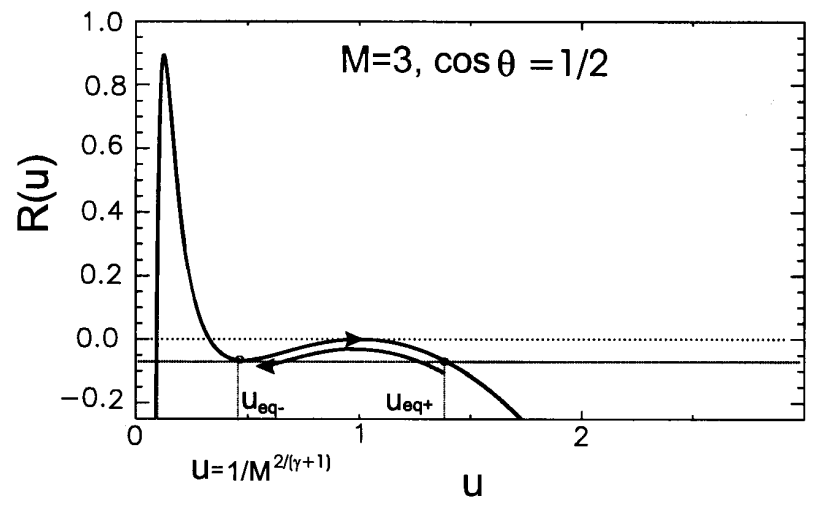

Fig. 3. $R(u)$ as a function of $u$ for $\gamma=2$ and $1>M>\cos \theta$. Since $R(u)$ has a double negative zero at the initial point, periodic waves may be constructed with amplitudes given by the intersections with the horizontal line, representing the initial driver energy, and the system oscillates between $u_{e q-}$ (compressive) and $u_{e q+}$ (rarefactive). There exists a critical driver, at which $u_{e q-}$ becomes sonic.

corresponds to the sonic point, $u_{i x}=c$, and the third at $u_{+}$, which is the only positive root of the equation

$\frac{M^{2}}{\cos ^{2} \theta}=\frac{u^{\gamma}-1}{\gamma u^{\gamma}(u-1)}$,

which follows from $\partial R / \partial u=0$. It follows that if $1>M>$ $\cos \theta, u_{+}<1$ and $R(u)$ has a double positive zero at $u=1$ (a prerequisite for soliton formation). The form of $R(u)$ is shown in Fig. 1, in which there are two other zeros of $R(u)$, one in the compressive region $u<1$ and the other in the rarefactive region $u>1$, but lying beyond the sonic point $u=1 / M^{[2 / \gamma+1]}$. Therefore, compressive solitons may be constructed with amplitude (centre of the wave) given by the compressive root of $R(u)=0$, which lies to the left of $u_{+}$. Although $R(u)$ possesses a rarefactive root, no rarefactive solitons can be constructed because, before reaching this possible equilibrium, the flow speed must go through the sound speed where the flow becomes choked $(d u / d x=\infty)$. The strength of compressive solitons, $u_{e q}$ (or $\phi_{m}$ ), given by $R(u)=0$ as a function of obliquity, is shown in Fig. 2 for various Mach numbers. The relation between the equilibrium compression $\left(u_{e q}\right)$ and its associated potential $\left(\phi_{m}\right)$ is given by the electron enthalpy, which in normalized form is

$\frac{1}{\left(\gamma_{e}-1\right) M_{e}^{2}}\left(\frac{1}{u^{\gamma_{e}-1}}\right)=\phi$.

If the wave speed is super-ion-acoustic $(M>1)$ the energy function $R(u)$ has a double negative zero and takes the form shown in Fig. 3. In this case we can construct periodic cnoidal like waves by adding a constant to right hand side of the wave structure equation. This constant represents the kinetic energy of some initial driver. Therefore, the intersections of a horizontal line, representing the initial driving energy, with the energy function $R(u)$, as illustrated in Fig. 3, gives the magnitudes of the compressive $(u<1)$ and rarefactive $(u>1)$ phases of the corresponding periodic wave.

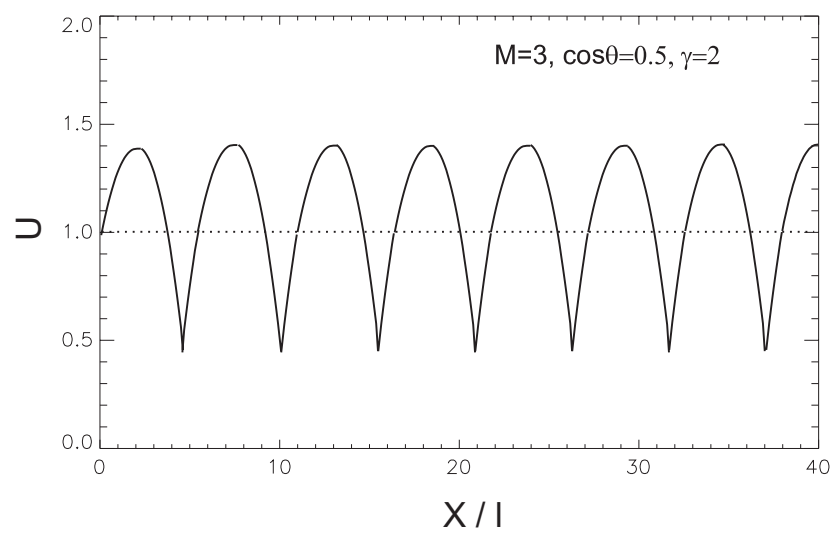

Fig. 4. Spiky periodic EICA waves for $u$ as a function of $x / l$, for the same parameters $(M$ and $\cos \theta)$ as used for Fig. 2.

Note that in this case, $M>1$, and the sonic point lies in the compressive phase of such a periodic wave, so that the flow is being driven towards this point, with the result that the slope $d u / d x$ is enhanced in the compressive phase. Thus there is a critical value of the driver constant such that its horizontal line touches $R(u)$ in the compressive range at the sonic point with the result that the waveform develops a cusp $(d u / d x \rightarrow \infty)$ at that point. Hence the periodic structure for the flow speed $u$ and the associated potential $\phi$ takes the forms depicted in Fig. 4. Periodic cusp shaped waveforms can, therefore, be generated by this trans-sonic feature where the flow becomes choked.

\section{Discussion}

We have shown that compressive soliton pulses propagate at subsonic speeds $(M<1)$ provided that the wave is sufficiently oblique, i.e. $\theta>\cos ^{-1} M$. In the weakly nonlinear limit $(|u-1|<<1)$ the wave structure Eq. (17) goes over to the stationary version of the $\mathrm{KdV}$ equation, and admits the classical sech ${ }^{2}$ hump of compression

$u=1+\delta_{m} \operatorname{sech}^{2}\left(\frac{x}{2 l}\right)$,

$$
\begin{aligned}
\delta_{m} & =-\frac{\left(M^{2} \cos ^{2} \theta-1\right)\left(1-M^{2}\right)}{(\gamma+1) M^{2}} \\
& \times\left[\cos ^{2} \theta\left(\frac{2 / 3}{M^{2}-1 / 3}\right)\right]
\end{aligned}
$$

where $\delta_{m}<0$ when $1>M>\cos \theta$. From this result it would also appear that solitons are restricted to a cone of obliquity

$M>\cos \theta>\cos \theta_{m}$,

$\cos \theta_{m}=\sqrt{\frac{2 / 3}{M^{2}-1 / 3}}$. 
However, this is a flaw of the weakly nonlinear approximation, since the fully nonlinear structure equation shows, in fact, that, provided $\theta>\cos ^{-1} M$, all subsonic oblique waves form soliton pulses with the greatest amplitudes occurring for perpendicular propagation, with $u \rightarrow 0$, in the fashion

$u \propto\left[\frac{(\pi / 2-\theta)^{2}}{2 \gamma M^{2}}\right]^{1 / \gamma}, \quad \theta \rightarrow \frac{\pi}{2}$.

Figure 2 provides a pictorial vindication of this fully nonlinear result. Note that in the laboratory frame these subsonic stationary waves correspond to wave frequencies in the evanescent gap, $\Omega \cos \theta<\omega<\Omega_{m}$, in which linear waves cannot propagate. On the other hand, if the wave speed is "supersonic", the wave frequencies in the laboratory frame, lie above the ion cyclotron cut-off frequency, in which nonlinear cnoidal waves can be generated by some initial driver. In this case the waveforms are asymmetric in nature with the compressive phase having sharper slopes than the rarefactive phases. In fact, if the driver is "critical", the end point of the compressive phase becomes sonic and the waveforms develop a cusp shape, as illustrated in Fig. 4. At this sonic point the potential $\phi$ is peaked with amplitude

$$
\begin{aligned}
& \frac{e \phi}{k T_{e}}=\frac{\gamma}{\gamma-1}\left[M^{\frac{2(\gamma-1)}{(\gamma+1)}}-1\right] \\
& =\ln (M), \quad(\gamma=1) .
\end{aligned}
$$

It is possible, as Reddy et al. (2002) have indicated in their numerical solution of this problem, that this trans-sonic feature may provide an explanation for the "spiky" waveforms observed in auroral electric fields (Ergun et al., 1998).
Acknowledgements. The authors wish to thank the National Research Foundation of South Africa (under grant number 2053776) and the University of KwaZulu-Natal for supporting this work, and the ISSI, Bern, Switzerland, where this work was presented at a workshop in March 2004.

Edited by: G. P. Zank

Reviewed by: two referees

\section{References}

Stix, T. H.: Waves in Plasmas, AIP, New York, 1992.

Temerin, M., Wolderif, M., and Mozer, P. S.: Nonlinear Steepening of Electrostatic Ion Cyclotron Waves, Phys. Rev. Lett., 43, 19411943, 1979.

Yu, M. Y., Shukla, P. K., and Bujarbarua, S.: Fully nonlinear ionacoustic waves in a magnetized plasma, Phys. Fluids, 23, 21462147, 1980.

Jovanic, P. and Shukla, P. K.: Nonlinear Model for Coherent Electric Field Structures in the Magnetosphere, Phys. Rev. Lett., 84, 4373-4376, 2000.

Reddy, R. V., Lakhina, G. S., Singh, N., and Bharuthram, R.: Spiky parallel electrostatic ion cyclotron and acoustic waves, Nonlin. Proc. Geophys, 9, 25-29, 2002,

SRef-ID: 1607-7946/npg/2002-9-25.

Andre, M., Koskinen, H., Gustafason, G. and Lundin, R.: Ion waves and upgoing ion beams observed by the Viking satellite, Geophys. Res. Lett., 14, 463-466, 1987.

Ergun, R., Carlson, B, McFadden, M.: FAST satellite observations of electric field structures in the auroral zone, Geophys. Res. Lett., 25, 2025-2028, 1998.

McKenzie J.F.: Nonlinear stationary waves of the ion-cyclotron type, Solitons and spiky waveforms, J. Plasma Phys., in press, 2004. 